\title{
The Clinical Outcomes of Transforaminal Percutaneous Endoscopic Discectomy in Treating Lumbar Disc Herniation: A Review
}

\author{
Bheemasetty Rakesh, Yun Tao Wang* \\ Department of Orthopedic Spine Surgery, Zhongda Hospital, Southeast University, Nanjing, China \\ Email:*wangyttod@aliyun.com
}

How to cite this paper: Rakesh, B. and Wang, Y.T. (2018) The Clinical Outcomes of Transforaminal Percutaneous Endoscopic Discectomy in Treating Lumbar Disc Herniation: A Review. Open Journal of Orthopedics, 8, 57-66.

https://doi.org/10.4236/ojo.2018.82008

Received: January 3, 2018

Accepted: February 9, 2018

Published: February 12, 2018

Copyright $\odot 2018$ by authors and Scientific Research Publishing Inc. This work is licensed under the Creative Commons Attribution International License (CC BY 4.0).

http://creativecommons.org/licenses/by/4.0/

(c) (i) Open Access

\begin{abstract}
Percutaneous endoscopic lumbar discectomy (PELD) is a minimally invasive technique started during the late $20^{\text {th }}$ century. This process is done through microscopic view under local anesthesia. There is a growing but still insufficient evidence that lumbar EDS shows slightly better results in terms of minor tissue damage, shorter hospital stay, faster return to ordinary daily activities, and patient satisfaction. Recurrence rate still remains a matter of debate, and is related with the surgical skills of the surgeon. The complication rate seems to be similar in both of the techniques i.e., open and endoscopic. More randomized controlled trials, systematic reviews and meta-analysis are needed to clarify whether lumbar EDS can be considered comparable if not superior to standard open discectomy. In spite of lacking defined clinical evidence, lumbar EDS is without doubt a rapidly expanding PELD and its future developments are incredibly promising. Due to less complication rate this technique can be considered as a gold standard compared to the open discectomy. The surgeons still require more cadaveric practices for learning the curve and to approach the herniated disc area. The main objective of this review article is to show the clinical outcomes of the Transforaminal Percutaneous Endoscopic Discectomy in treating the lumbar disc herniation.
\end{abstract}

\section{Keywords}

Endoscopic Visualization, Intervertebral Disc, Fluoroscope Guidance, Selective Chromoendoscopy, Intervertebral Nucleotomy, Lumbar Disc Herniation

\section{Introduction}

Lumbar disc herniation (LDH), is one of the important reasons behind the nerve 
root pain which severely affects the quality of life of the adults. LDH is the herniation of the soft central portion (nucleus pulposus) which is located in the middle of the outer fibrous ring (annulus fibrosus) of the intervertebral disc which leads to severe lower back pain, numbness or weakness, sciatica and sometimes leads to paralysis or bladder incontinence. The Figure 1 shows the clear anatomical view of the normal lumbar disc and the herniated lumbar disc.

Earliest successions of Endoscopic discectomy are reported after the late '80s. Kambin and Schaer reported their successive treatment in $88 \%$ of patients who underwent percutaneous discectomy [1] and the related outcomes after the introduction of the minimally invasive technique so called arthroscopic discectomy [2]. In the late ' 80 s and the beginning of the ' 90 s other authors stated analogous outcomes [3] [4] [5] [6] [7]. It is reported as a technique with a far lateral or posterolateral approach to disc through the lateral foramen. This surgery is performed under radiological guidance, endoscopically introduced cannulated system for the herniated nucleus pulposus fragment removal.

Consequential combination of the procedures headed to the extension of reports in the mid ' $90 \mathrm{~s}$. With the developing surgical proficiency, the far lateral approach was criticized by many professionals. In $7 \%$ to $11 \%$ of the cases, patients underwent re-exploration surgery because of the lack of radicular pain relief [8] [9] [10] [11]. In a relative review Kim and Park mentioned that the percutaneous discectomy is limited to anatomical factors, such as iliac crest, large facet joint, or L5 transverse process [12]. To get rid of these problems, endoscopic interlaminar approach was consequently developed and popularized by many professionals [13] [14] [15] [16]. Numerous studies have been published regarding the endoscopic discectomy compared with the microscopically open surgeries and open lumbar discectomy discussed in the clinical outcomes. In the Figure 2 we can see the herniated lumbar disc at $14-15$ level and the spinal nerve compressed by the herniated nucleus pulposus.

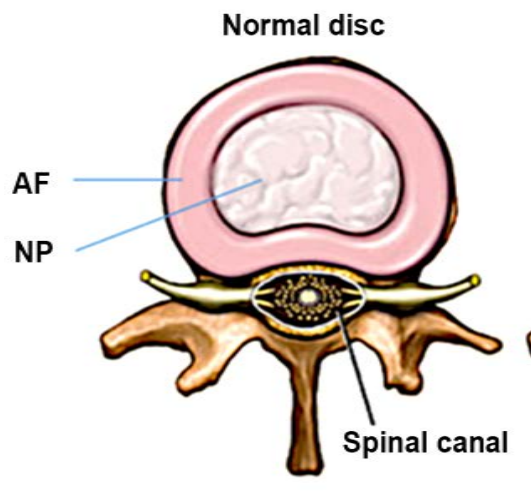

(a)

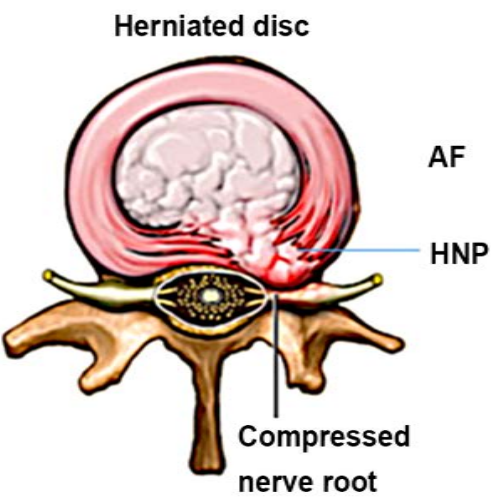

(b)

Figure 1. Anatomical view of the lumbar disc (a) normal disc showing the annulus fibrosus (AF), nucleus pulpous (NP), spinal nerve and (b) herniated disc showing the herniation of the nucleus pulposus leading to the compression of the nerve root. 


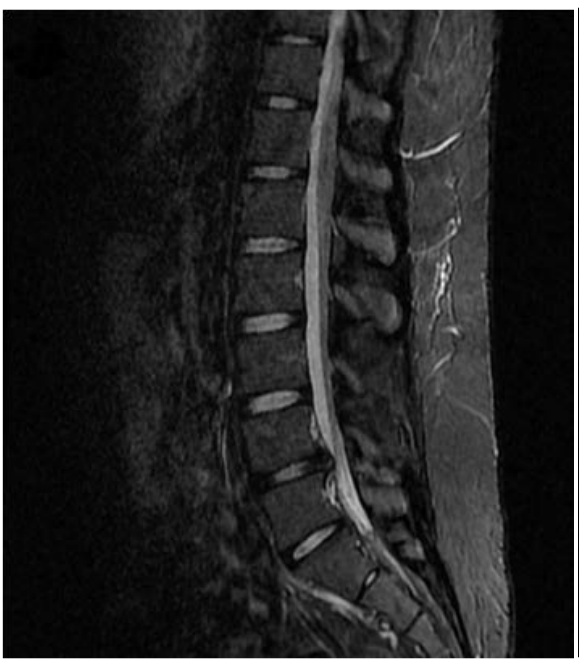

(a)

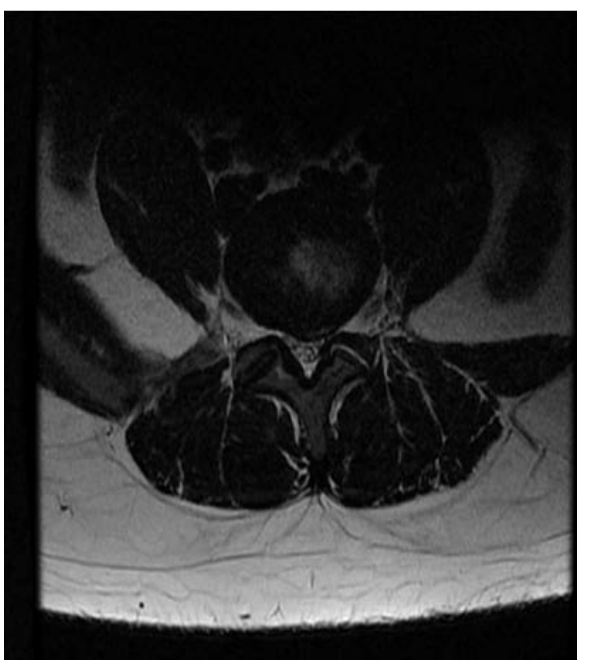

(b)

Figure 2. (a) Magnetic resonance image (MRI) of the herniated nucleus pulposus at L4-L5 level; (b) compression of the spinal nerve.

\section{Surgical Technique}

As stated above, Endoscopic discectomy has been widely practiced, and many changes in the procedures were reported. However, to summarize we can say that endoscopic discectomy has two different approaches: the first one being the transforaminal approach; possible variations of this name include far lateral endoscopic approach, posterior-lateral endoscopic approach, and arthroscopic far lateral/posterior-lateral approach. The second one is the interlaminar approach as described by Ruetten et al. [14]. Indications and procedures for these approaches are different, and both need thorough postop assessments. The Figure 3 explains the transforaminal approach to the herniated disc and removal of the herniated nucleus pulposus.

\section{Transforaminal Approach:}

Indications: Patients complained with severe lower back pain which is non-responsive to epidural steroidal injection, to remove the inflammatory section that causes discogenic pain and to decompress the compressed nerve components.

Contraindications: L5-S1 segment (iliac crest and/or L5 transverse process are obstacles for surgical route), anatomical variations, large median and para median disc herniation/cauda equina syndrome, Spinal stenosis, post laminectomy recurrent herniation.

Advantages: It's a minimally invasive approach, there is lower degree of muscle manipulation/damage, reduced low back pain after surgery, reduced postop fibrosis (both muscle and periradicular), and limited bone decompression prevent risk of postop instability due to excessive removal of facet joint, direct visualization of decompressed root from its extracanalar route.

Disadvantages: Lack of experience and learning curve for surgeons who are used to standard micro-discectomy, gradu0ally more limited movements as the 


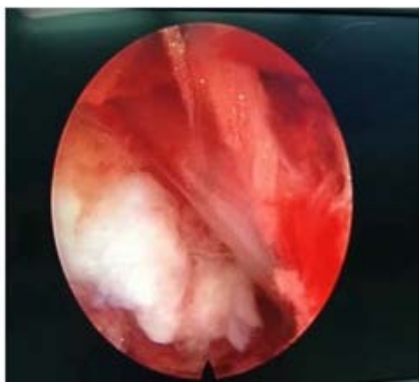

(a)

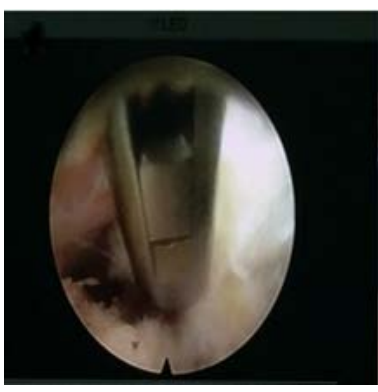

(b)

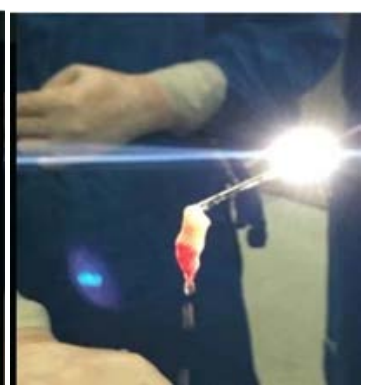

(c)

Figure 3. Transforaminal endoscopic discectomy. (a) shows the nerve root and the herniated disc; (b) removal of the herniated disc; (c) herniated nucleus pulposus.

foramen is entered, and no possible treatment for L5-S1 level and median disc herniation.

\section{Post-Surgical Schedule}

It being a minimally invasive procedure the patient can walk after $1-2 \mathrm{hr}$ of the surgery. However, during local anesthesia, the patient sometimes experiences numbness because of lidocaine infiltrating the exiting nerve. Bed rest is advised in this case. Patients can be discharged in 1 to 3 days prior to the surgery. He/she is allowed to do office work after 4 - 5 days, and they are not allowed to lift heavy weights for $6-8$ weeks to avoid recurrence of the herniation. The patient can take up sports 5 - 6 weeks after the surgery in most of the cases but contact sports should carefully be determined.

\section{Surgical Indication}

Far-lateral HNP: Direct insertion of the cannula can be done into the HNP for those located outside the canal. This makes it relatively easy to remove the mass avoiding the need of insertion of the cannula through the intervertebral foramen. Under local anesthesia, a far lateral HNP can be removed at every lumbar levels by the posterolateral approach [17] [18].

Recurrent HNP: There are scar tissues and adhesions around the nerve root. Because of this reason, it is technically demanding to safely remove a recurring HNP at the same level by Love's or micro Love's technique or MED. For PED, the transforaminal approach can be used as the initial operation. Based on their experience of 463 cases [19], Ruetten et al. commented on the usefulness of PED for recurrent HNP and consequently reported its utility [20].

\section{Clinical Outcome}

Numerous studies have been published regarding the endoscopic discectomy compared with the microscopically open surgeries and open lumbar discectomy. Among all the reviews published recently Dorham and Mansour showed an outstanding report of the outcomes between the microdiscectomy and the endoscopic discectomy which are surprisingly so similar [21]. The main drawback of 
their review was they don't explain about the recurrence rate and complications. The main drawback behind the clarification of the endoscopic discectomy as a standard technique to lack of proper RCT'S and proper comparisons between the two micro and the endoscopic techniques.

For classifying the clinical outcomes between the two micro and endoscopic discectomy so many studies were analyzed by smith and his teammates and among them only 4 RCT's matched with the criteria which is eligible to compare the two techniques and the results were not up to the expectations [22]-[27] Teli et al., published on of the largest randomized study and reported so many clinical outcomes with many complications regarding the ED (endoscopic discectomy).

Precisely Rasouli et al. [28], did a detailed RCT comparing all minimally invasive surgical techniques in which he elaborated all the clinical outcomes of the endoscopic discectomy includes the pain index, recurrence rate, complications and the postoperative hospital stay and the major risk factors. Overall one meta-analysis was reported with 9 RCT's comparing all the above mentioned outcomes by using the VAS, ODI and the MacNab which showed a good significant statistical analysis in regarding the risk factors like neural weakness, blood loss [29]. Although the results of this meta-analysis is very satisfying still many authors needs more clinically done randomized controlled trials for future improvements and to make the endoscopic discectomy as the standard procedure for the lumbar disc herniation.

The re-herniation rate of $20 \%$ in patients underwent transforaminal approach endoscopic discectomy in treating LDH was reported [30], and similar results was stated by Wang et al. in a study relating two different surgeons in learning the curve [31]. Complications including the dysesthesia, CSF leak, nerve root damage, etc. are quite identical in all series.

The first clinically evaluated outcomes of the endoscopic discectomy were done by Yeung and Tsou [32]. It showed a highest satisfactory result among all the patients and it is almost equaled the outcome rate compared to the open surgeries. Many comparisons were made by many authors and among them Ruetten et al. reported the same results compared with the micro and endoscopic discectomy and thee study shows $82 \%$ of the patients did not resulted in the postoperative leg pain and $12 \%$ resulted in intermittent pain.

Birkenmaier et al. made five comparative studies of the present technique and conventional open surgery and came to the conclusion that PED had similar clinical outcomes. They also highlighted the advantages of PED which are as follows: shorter operating time, lower estimated blood loss during surgery, fewer surgery-related complications, less surgical site pain immediately after surgery, less need for postoperative pain medication, shorter hospital stay, and a faster return to work.

\section{Complications}

Complications should be primarily informed by the surgeon to the patient as a 
consent which are as follows 1) Unable to access to the area involved with a high iliac crest. 2) Lumbar plexopathy 3) Hematoma can occur in the subcutaneous tissue or between the psoas major and minor muscles superficially or deep layered [33].

The complications which were observed during and after PELD with the transforaminal approach includes: 1) Exiting nerve root injury 2) Dural tear 3) Infection near the surgical site 3) Cauda equina injury 4) Hematoma 5) Vessel injury.

Surgeons should be very much attentive regarding the intracranial hypertension while performing surgery because it might result in headaches, seizures and even death. Choi et al. reported 4 seizure cases [4]. The patients who are observed with seizures complained of neck pain, so the neck pain was considered as the most possible sign of the subsequent seizure.

\section{Future Perspectives}

PELD technique can be done by laminectomy with a high-speed drill for lumbar spinal canal stenosis [34] and by radiofrequency thermal annuloplasty for low back pain [35]. These techniques are not widely in use so more clinical trials and more technical seminars and laboratory experiments should be done on fresh cadavers for future benefits and clear details of these advanced techniques.

\section{Discussion}

For highly migrated disc herniation, PELD in transforaminal approach is still challenging due to the limited view and ease of access to the target fragment. Therefore, for highly migrated herniations open surgery was recommended. This review has some major limitations and, as stated earlier, our goal was to provide an update about this topic, focusing on the current issues (recurrence/complications rate) and on possible future advances. At present the number of centers and surgeons practicing EDS is exponentially increasing [36]. Considering from the early ' 90 s, in the last decade there is a rapid increase in the application of EDS. 51 surgical series were reported in the English literature, and far more were found in other languages. Moreover, our focus was only on transforaminal endoscopic discectomy, excluding recurrence series and series focused on a specific aspect.

In 2015 one of the largest series of EDS reported was published which included 10228 patients treated through a transforaminal approach [37]. In 2.8\% of the cases, the authors reported an incomplete removal and recurrence rate of $0.8 \%$; both of these two types of data are comparable to those reported on standard microdiscectomy series. Amazingly, the authors determined their focus on the rate of incomplete removal and recurrences related to the learning curve of the surgeons and the inappropriate positioning of the surgical equipments, which were found to be the main causes of negative outcome in this particular study [38]. 
The vital importance for the outcome is appropriate indications. According to the authors' experience and basis of the literature data, these procedures are applicable in patients observed fresh or relatively fresh fragments, even migrated, with minor or no signs of diffuse spinal degenerative disease, such as broad disc bulging, spinal stenosis secondary to hypertrophic ligament/osteophytes, and spinal instability [39]. Additionally, for surgeons not used to the techniques and endoscopic kit, use of the endoscope in spinal procedures may be very challenging and it requires training and learning curve. Two series which were recently reported highlighted the results obtained from surgeons with varying level of experience in EDS. In particular, both of these articles reported higher rate of recurrence/residual in patients who were operated by surgeons at the earlier stage of their learning curve [40].

Lack of RCT's keeps us wary about the clarification of these outcomes. Preferably, a multi-centered RCT's enrolling huge number of patients and surgeons with similar degree of experience should elucidate whether results of EDS are similar or better than the ones with standard micro-discectomy.

\section{Conclusions}

In spite of lacking defined clinical evidence, lumbar EDS is without doubt a rapidly expanding PELD and its future developments are incredibly promising. Although it's not mentioned here, applications of endoscopic techniques are gradually increasing to other lumbar diseases, such as instability, [41] [42] multi levels surgery, [43] recurrent discs [44], and spinal stenosis [45] [46].

Based on the available data about lumbar EDS, following points are highlighted.

1) There is a growing but still insufficient evidence that lumbar EDS shows slightly better results in terms of minor tissue damage, shorter hospital stay, faster return to ordinary daily activities, and patient satisfaction.

2) Rate of recurrence/residual still remains a matter of debate, and it seems to be strictly related to appropriate surgical indications and level of training of the surgeon.

3) In both open and endoscopic techniques, rate of complications seems similar; however the reported results are enormously nonhomogeneous in different series.

4) More randomized controlled trials, systematic reviews and meta-analysis are needed to clarify whether lumbar EDS can be considered comparable if not superior to standard open discectomy.

\section{References}

[1] Kambin, P. and Schaffer, J.L. (1989) Percutaneous Lumbar Discectomy: Review of 100 Patients and Current Practice. Clinical Orthopaedics and Related Research, 238, 24-34. https://doi.org/10.1097/00003086-198901000-00004

[2] Kambin, P. (1992) Arthroscopic Microdiscectomy. Arthroscopy. The Journal of Arthroscopic \& Related Surgery, 8, 287-295. 
https://doi.org/10.1016/0749-8063(92)90058-J

[3] Kovac, D. (1990) Automated Endoscopic Percutaneous Diskectomy in the Treatment of Lumbar Disk Hernia. Lijecnicki vjesnik, 113, 158-161.

[4] Leu, H. and Schreiber, A. (1989) 10 Years of Percutaneous Disk Surgery: Results and Developments. Schweizerische Rundschau fur Medizin Praxis = Revue suisse de medecine Praxis, 78, 1434-1439.

[5] Suezawa, Y. and Schreiber, A. (1987) Percutaneous Nucleotomy with Discoscopy. 7 Years' Experience and Results. Zeitschrift fur Orthopadie und ihre Grenzgebiete, 126, 1-7. https://doi.org/10.1055/s-2008-1044858

[6] Schreiber, A. and Suezawa, Y. (1986) Transdiscoscopic Percutaneous Nucleotomy in Disk Herniation. Orthopaedic Review, 15, 35-38.

[7] Hausmann, B. and Forst, R. (1985) Nucleoscope. Instrumentarium for Endoscopy of the Intervertebral Disc Space. Archives of Orthopaedic and Traumatic Surgery, 102, 57-59. https://doi.org/10.1007/BF00443041

[8] Kambin, P., et al. (1998) Arthroscopic Microdiscectomy and Selective Fragmentectomy. Clinical Orthopaedics and Related Research, 347, 150-167. https://doi.org/10.1097/00003086-199802000-00018

[9] Kambin, P. and Sampson, S. (1986) Posterolateral Percutaneous Suction-Excision of Herniated Lumbar Intervertebral Discs: Report of Interim Results. Clinical Orthopaedics and Related Research, 207, 37-43.

https://doi.org/10.1097/00003086-198606000-00008

[10] Tsou, P.M. and Yeung, A.T. (2002) Transforaminal Endoscopic Decompression for Radiculopathy Secondary to Intracanal Noncontained Lumbar Disc Herniations: Outcome and Technique. The Spine Journal, 2, 41-48. https://doi.org/10.1016/S1529-9430(01)00153-X

[11] Yeung, A.T. and Tsou, P.M. (2002) Posterolateral Endoscopic Excision for Lumbar Disc Herniation: Surgical Technique, Outcome, and Complications in 307 Consecutive Cases. Spine, 27, 722-731. https://doi.org/10.1097/00007632-200204010-00009

[12] Kim, H.S. and Park, J.Y. (2013) Comparative Assessment of Different Percutaneous Endoscopic Interlaminar Lumbar Discectomy (PEID) Techniques. Pain Physician, 16, 359-367.

[13] Choi, G., et al. (2006) Percutaneous Endoscopic Interlaminar Discectomy for Intracanalicular Disc Herniations at L5-S1 using a Rigid Working Channel Endoscope. Operative Neurosurgery, 58, ONS5968.

[14] Ruetten, S., Komp, M. and Godolias, G. (2006) A New Full-Endoscopic Technique for the Interlaminar Operation of Lumbar Disc Herniations using 6-mm Endoscopes: Prospective 2-Year Results of 331 Patients. Minimally Invasive Neurosurgery, 49, 80-87. https://doi.org/10.1055/s-2006-932172

[15] Ruetten, S., et al. (2007) Use of Newly Developed Instruments and Endoscopes: Full-Endoscopic Resection of Lumbar Disc Herniations via the Interlaminar and Lateral Transforaminal Approach. Journal of Neurosurgery: Spine, 6, 521-530.

[16] Ruetten, S., et al. (2008) Full-Endoscopic Interlaminar and Transforaminal Lumbar Discectomy versus Conventional Microsurgical Technique: A Prospective, Randomized, Controlled Study. Spine, 33, 931-939. https://doi.org/10.1097/BRS.0b013e31816c8af7

[17] Mayer, H.M. (2006) Minimally Invasive Spine Surgery. In: Mayer, M., Ed., Minimally Invasive Spine Surgery, Springer, Berlin, 3-7.

[18] Lee, S.-H., et al. (2006) Operative Failure of Percutaneous Endoscopic Lumbar Dis- 
cectomy: A Radiologic Analysis of 55 Cases. Spine, 31, E285-E290.

[19] Peterson, R. (1991) Posterolateral Microdiskectomy in a General Orthopaedic Practice. Seminars in Orthodontics, 6, 117.

[20] Lee, S., et al. (2007) Percutaneous Endoscopic Lumbar Discectomy for Migrated Disc Herniation: Classification of Disc Migration and Surgical Approaches. European Spine Journal, 16, 431-437. https://doi.org/10.1007/s00586-006-0219-4

[21] Dohrmann, G.J. and Mansour, N. (2015) Long-Term Results of Various Operations for Lumbar Disc Herniation: Analysis of over 39,000 Patients. Medical Principles and Practice, 24, 285-290. https://doi.org/10.1159/000375499

[22] Smith, N., et al. (2013) Systematic Review of Microendoscopic Discectomy for Lumbar Disc Herniation. European Spine Journal, 22, 2458-2465. https://doi.org/10.1007/s00586-013-2848-8

[23] Birkenmaier, C., et al. (2013) The Current State of Endoscopic Disc Surgery: Review of Controlled Studies Comparing Full-Endoscopic Procedures for Disc Herniations to Standard Procedures. Pain Physician, 16, 335-344.

[24] Garg, B., Nagraja, U.B. and Jayaswal, A. (2011) Microendoscopic versus Open Discectomy for Lumbar Disc Herniation: A Prospective Randomised Study. Journal of Orthopaedic Surgery, 19, 30-34. https://doi.org/10.1177/230949901101900107

[25] Teli, M., et al. (2010) Higher Risk of Dural Tears and Recurrent Herniation with Lumbar Micro-Endoscopic Discectomy. European Spine Journal, 19, 443-450. https://doi.org/10.1007/s00586-010-1290-4

[26] Huang, T.J., et al. (2005) Less Systemic Cytokine Response in Patients Following Microendoscopic versus Open Lumbar Discectomy. Journal of Orthopaedic Research, 23, 406-411. https://doi.org/10.1016/j.orthres.2004.08.010

[27] Righesso, O., Falavigna, A. and Avanzi, O. (2007) Comparison of Open Discectomy with Microendoscopic Discectomy in Lumbar Disc Herniations: Results of a Randomized Controlled Trial. Neurosurgery, 61, 545-549. https://doi.org/10.1227/01.NEU.0000290901.00320.F5

[28] Rasouli, M.R., et al. (2013) Minimally Invasive Discectomy versus Microdiscectomy/Open Discectomy for Symptomatic Lumbar Disc Herniation. The Cochrane Database of Systematic Reviews, No. 9, CD010328.

[29] Cong, L., Zhu, Y. and Tu, G. (2016) A Meta-Analysis of Endoscopic Discectomy versus Open Discectomy for Symptomatic Lumbar Disk Herniation. European Spine Journal, 25, 134-143. https://doi.org/10.1007/s00586-015-3776-6

[30] Tenenbaum, S., et al. (2011) Percutaneous Posterolateral Transforaminal Endoscopic Discectomy: Clinical Outcome, Complications, and Learning Curve Evaluation. Surgical Technology International, 21, 278-283.

[31] Wang, B., et al. (2011) An Evaluation of the Learning Curve for a Complex Surgical Technique: The Full Endoscopic Interlaminar Approach for Lumbar Disc Herniations. The Spine Journal, 11, 122-130. https://doi.org/10.1016/j.spinee.2010.12.006

[32] Tsou, P.M., Yeung, C.A. and Yeung, A.T. (2004) Posterolateral Transforaminal Selective Endoscopic Discectomy and Thermal Annuloplasty for Chronic Lumbar Discogenic Pain: A Minimal Access Visualized Intradiscal Surgical Procedure. The Spine Journal, 4, 564-573. https://doi.org/10.1016/j.spinee.2004.01.014

[33] Wang, H., et al. (2015) Risk Factors for Failure of Single-Level Percutaneous Endoscopic Lumbar Discectomy. Journal of Neurosurgery: Spine, 23, 320-325. https://doi.org/10.3171/2014.10.SPINE1442

[34] Ruetten, S., et al. (2013) Decompression of Lumbar Lateral Spinal Stenosis: 
Full-Endoscopic, Interlaminar Technique. Operative Orthopadie und Traumatologie, 25, 31-46. https://doi.org/10.1007/s00064-012-0195-2

[35] Sairyo, K., Kitagawa, Y. and Dezawa, A. (2013) Percutaneous Endoscopic Discectomy and Thermal Annuloplasty for Professional Athletes. Asian Journal of Endoscopic Surgery, 6, 292-297. https://doi.org/10.1111/ases.12055

[36] Son, I.-N., Kim, Y.-H. and Ha, K.-Y. (2015) Long-Term Clinical Outcomes and Radiological Findings and Their Correlation with Each Other after Standard Open Discectomy for Lumbar Disc Herniation. Journal of Neurosurgery: Spine, 22, 179-184. https://doi.org/10.3171/2014.10.SPINE131126

[37] Choi, K.-C., et al. (2015) Unsuccessful Percutaneous Endoscopic Lumbar Discectomy: A Single-Center Experience of 10228 Cases. Neurosurgery, 76, 372-381. https://doi.org/10.1227/NEU.0000000000000628

[38] Yorimitsu, E., et al. (2001) Long-Term Outcomes of Standard Discectomy for Lumbar Disc Herniation: A Follow-Up Study of More than 10 Years. Spine, 26, 652-657. https://doi.org/10.1097/00007632-200103150-00019

[39] Lee, D.Y. and Lee, S.-H. (2008) Learning Curve for Percutaneous Endoscopic Lumbar Discectomy. Neurologia Medico-Chirurgica, 48, 383-389.

https://doi.org/10.2176/nmc.48.383

[40] Chaichankul, C., Poopitaya, S. and Tassanawipas, W. (2012) The Effect of Learning Curve on the Results of Percutaneous Transforaminal Endoscopic Lumbar Discectomy. Journal of the Medical Association of Thailand, 95, S206-S212.

[41] Jasper, G.P., Francisco, G.M. and Telfeian, A.E. (2013) Clinical Success of Transforaminal Endoscopic Discectomy with Foraminotomy: A Retrospective Evaluation. Clinical Neurology and Neurosurgery, 115, 1961-1965. https://doi.org/10.1016/j.clineuro.2013.05.033

[42] Telfeian, A.E. (2014) Transforaminal Endoscopic Discectomy with Foraminoplasty for the Treatment of Spondylolisthesis. Pain Physician Journal, 17, E703-E708.

[43] Hur, J.-W., et al. (2014) Percutaneous Endoscopic Lumbar Discectomy and Annuloplasty for Lumbar Disc Herniation at the Low Two Contiguous Levels: Single-Portal, Double Surgeries. Journal of Neurological Surgery Part A: Central European Neurosurgery, 75, 381-385.

[44] Nomura, K., et al. (2014) A Novel Microendoscopically Assisted Approach for the Treatment of Recurrent Lumbar Disc Herniation: Transosseous Discectomy Surgery. Journal of Neurological Surgery Part A: Central European Neurosurgery, 75, 183-188.

[45] Ahn, Y. (2014) Percutaneous Endoscopic Decompression for Lumbar Spinal Stenosis. Expert Review of Medical Devices, 11, 605-616. https://doi.org/10.1586/17434440.2014.940314

[46] Xu, B.S., et al. (2010) Bilateral Decompression via Unilateral Fenestration Using Mobile Microendoscopic Discectomy Technique for Lumbar Spinal Stenosis. Orthopaedic Surgery, 2, 106-110. https://doi.org/10.1111/j.1757-7861.2010.00072.x 